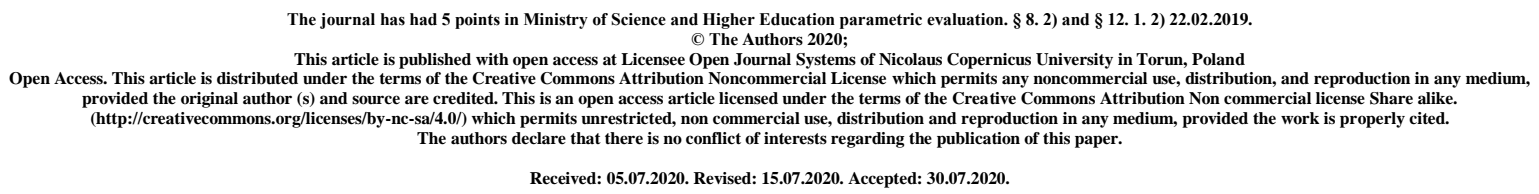

\title{
Nanoparticles in medicine
}

\author{
Paulina Miziak, Marzena Baran', Katarzyna Bonio², Piotr Piech ${ }^{3}$ \\ ${ }^{1}$ Chair and Department of Biochemistry and Molecular Biology, Medical University of Lublin \\ ${ }^{2}$ Department of Cell Biology, Institute of Biological Sciences, Maria Curie-Skłodowska University, Lublin \\ ${ }^{3}$ Human Anatomy Department, Medical University of Lublin, Poland
}

\begin{abstract}
Nanotechnology is a new, interdisciplinary field of science, the products of which are nanoparticles, i.e. structures that, thanks to their unique physical and chemical properties conditioned by their nanometric dimensions, have found wide application in medicine, pharmacy and biotechnology. Currently, they play a particularly important role as drug carriers, antibacterial and antiviral substances. They are also used in diagnostics, genetic tests, and increasing the accuracy of biological tests. The enormous possibilities of nanoparticles concern their potential use as drug delivery systems, factors improving the quality of anticancer radiotherapy and supporting molecular imaging, as well as compounds with highly bactericidal, fungicidal and antiviral properties. Therefore, it is crucial to implement therapies based on nanoparticles into clinical trials, as they can be an excellent diagnostic and therapeutic tool for many diseases, including cancer. However, it is necessary to overcome certain barriers due to the nature of some nanoparticles, such as problems with biodegradability and porosity. Despite the benefits of using nanostructures, the possibility of side effects caused by the action of metal nanoparticles absorbed by organisms has not been ruled out.
\end{abstract}

Key words: nanoparticle, medicine, drug delivery, peptide, tissue 


\section{Introduction}

Nanoparticles are powerful tools used for imaging, diagnostics and therapy, mainly due to their properties. They are of the same magnitude as antibodies, membrane receptors, nucleic acids, and proteins. They also have biomimetic features that, combined with miniaturization, make it possible to transform their applications [1]. However, the sheer size of nanoparticles is not enough to be used for imaging or diagnosis. For interaction with a biological target to occur, a molecular shell or layer that acts as a bioinorganic interface should be bonded to the nanoparticle. Examples of such coatings may be antibodies, biopolymers such as collagen, or single layers of small molecules, which make these particles biocompatible and ensure their proper functioning in a living organism [2]. Despite the great possibilities offered by nanoparticles, scientists are still working on using them in medicine, an example of which can be research on in vivo real-time monitoring of cellular processes, or specific targeting and delivery of drugs to the site of action in a target cell [1].

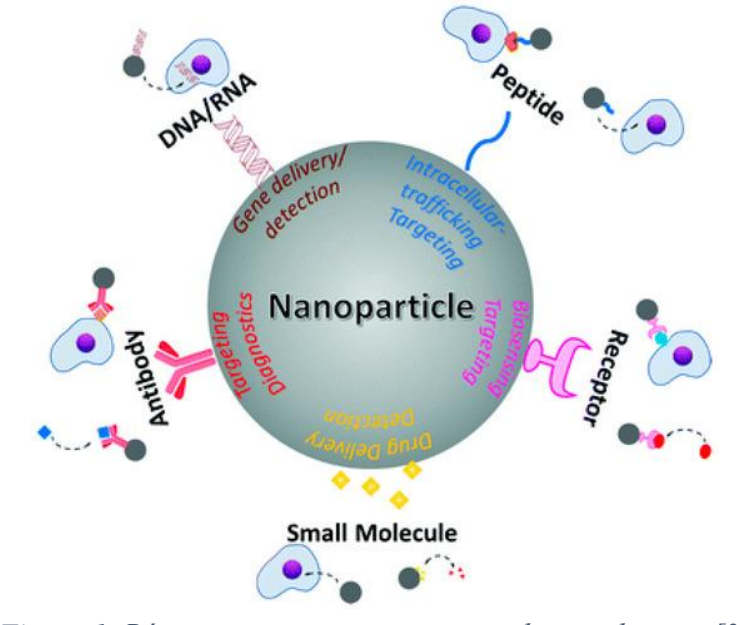

\section{Tissue engineering}

Figure 1. Różne zastosowania nanoczastek w medycynie [3]

Tissue engineering is a rapidly growing branch of science, so new tools are needed to better understand, evaluate and monitor new biomaterials used in targeted tissue growth. One of such biomaterials are carbon nanotubes. They can be used as a scaffold for tissue growth and facilitate the delivery of growth stimulating factors in the microenvironment. Due to the ability to conduct electricity, it is possible to direct cell growth by means of precisely defined electrical impulses. The use of these structures facilitates the assessment of tissue growth using magnetic resonance imaging, which facilitates modeling and planning for the use of new methods in tissue engineering. The cytotoxicity of carbon nanotubes can be easily eliminated by modifying and substituting functional groups on their surface [4]. The natural surface of the bone is not completely smooth, it contains natural cavities (pores) with a diameter of $100 \mathrm{~nm}$. This structure is important during implant transplantation. If the surface of the implant were completely smooth, the body would try to reject such a transplant. Due to the smooth surface it is highly likely that fibrous tissue will start to be produced covering the surface of the implant. This layer hinders (reduces) the contact of the graft with the recipient's bone, which may result in inflammation and dangerous pathogenic changes. It has been shown that creating nano-sized protrusions on the surface of the prosthesis may reduce the chances of rejection and stimulate the production of osteoblasts, increasing the chances of graft acceptance. Osteoblasts are cells responsible for the growth of the bone matrix, located 
on the surface of developing bone. Using nano-structured metal surfaces, it was observed that more than $90 \%$ of the bone cells adhered to them, which gives a noticeable increase over the control sample, where the number of adherent cells was only $50 \%$. These discoveries will allow the design of more durable and durable (longer-lasting) hip and knee implants and reduce the chances of rejection of the implants [5]. Titanium is a well-known material used in orthopedics and dentistry. It has high fracture resistance, high plasticity and a correspondingly low weight in relation to strength. The problem encountered when using this material is its lack of bioactivity, it does not support cell adhesion and growth. Apatite (a mineral from the phosphate cluster) covers, on the other hand, are bioactive and form a bond with the bone. This is due to their structure and similarity to the natural components of bone. In the past, techniques of covering titanium implants with apatite have been used to increase their bioactivity and reduce the risk of complications after transplantation. The disadvantage of these coatings was their heterogeneity, poor adhesion and low mechanical strength. It has been shown that the biomimetic approach - slow growth of the nanostructured apatite film from artificial body fluid - results in the formation of a strongly adherent uniform nanoporous layer [6]. This layer is made of $60 \mathrm{~nm}$ crystallites and has a stable nanoporous structure and bioactivity towards osteoblasts. Real bone is a nanocomposite material made of hydroxyapatite crystals embedded in a collagen organic matrix. As a result, the bone is mechanically hard and at the same time plastic, and has great regeneration abilities after mechanical damage [7].

\section{Anticancer therapy}

The term nanomedicine can be defined as those branches of science that use nanotechnologies in medicine. Nanoparticles can be used as drug carriers in cancer therapies. Such pharmaceutical carriers are passively targeted at neoplasms due to their increased ability to penetrate the cell membranes of cells, which means that chemotherapeutic agents can be selectively delivered to cancer cells [8]. Photodynamic anti-cancer therapy is based on the destruction of cancer cells by a laser that generates atomic oxygen, which is cytotoxic. A greater amount of special dye is taken to generate atomic oxygen in the case of cancer cells compared to healthy tissue. As a result, only cancer cells are destroyed and exposed to laser radiation. Unfortunately, the remnants of the periwinkle migrate into the skin and eyes of the patient, making them sensitive to daylight exposure. This effect can last up to 6 weeks. To avoid this side effect, the hydrophobic version of this dye has been closed inside the porous nanoparticle. The dye was trapped inside the ORMOSIL (organically modified silica) nanoparticle and did not spread to other parts of the body. At the same time, the ability to create oxygen was not affected, and the pore size of $1 \mathrm{~nm}$ freely allowed for oxygen diffusion to the outside [9].

\section{Detecting proteins}

Proteins are an essential element of cellular language, mechanism and structure, and understanding their functions is extremely important for the further development of the human body. Gold nanoparticles are commonly used for immunohistochemical analysis to identify protein-protein interactions. However, the simultaneous multi-channel detection capabilities of this technique are quite limited. Surface enhanced Raman spectroscopy is the 
best technique used to detect and identify selectively stained molecules. By combining both methods into single nanoparticle probes, the capabilities of multiplex protein probes can be significantly improved [10]. The group of prof. Mirkina has designed an advanced multifunctional probe that is built around $13 \mathrm{~nm}$ gold nanoparticles. The nanoparticles are coated with hydrophilic oligonucleotides having Raman dye at one end and end-capped with small particles for element/ element recognition. Moreover, this particle is catalytically active and coated with silver in a solution of $\mathrm{Ag}$ (I) and hydroquinone. Then, the probe is connected to a small molecule or antigen to be detected, the medium is exposed to the silver and hydroquinone solution. The silvering occurs near the Raman dye, which allows the detection of the dye signatures on a standard Raman microscope. In addition to recognizing small molecules, the probe can be modified to identify antibodies present on the surface of proteins. When examining the protein matrix, except for small particles and proteins, the probe showed no cross-reactivity [7].

\section{Drug delivery}

New methods of drug delivery are of wide interest to pharmaceutical companies. One of them is the use of nanotechnology as drug delivery systems. The large-scale application of these methods raises great hopes. However, in clinical trials, limitations are encountered due to their poor bio-pharmacological properties, such as low water solubility or limited intestinal epithelial permeability. These limitations result in poor drug bioavailability and undesirable pharmacokinetic properties. The development of technology for the synthesis of drugs free of the above-mentioned drawbacks may increase the durability and effectiveness of the product, which translates into the safety of their use in patients and ultimately may lead to a reduction in health care costs [11]. Controlled drug delivery systems (DDS) have many advantages over traditional forms of drug delivery in that the drug is transported to the site of action, thus minimizing side effects and their effects on residual tissues. Accumulation of therapeutic compounds at the target site is increased, resulting in lower drug doses required. This modern form of therapy is especially important when there is a discrepancy between the dose or concentration of the drug and its therapeutic or toxic effects [12]. Nanocarriers with optimized physicochemical and biological properties are taken up by cells more easily than larger molecules, so they can be used as delivery agents for currently available biologically active compounds. The method of attaching a drug to the nanocarrier and the strategy of targeting it is essential for targeted treatment. The drug may be adsorbed, covalently attached to the surface of the nanocarrier, or it may be encapsulated therein. Covalent bonding has the advantage over other connection methods in that it enables the control of the number of drug molecules associated with nanocarriers, i.e. precise control of the amount of therapeutic mixture delivered to a given tissue. Specific drug targeting with nanocarriers can be achieved by active or passive mechanisms. The first mechanism is to attach the drug to a specific part of the nanocarrier by means of appropriate ligands attached to the surface of the antibodies. Passive targeting is the result of the increased vascular permeability and retention that is characteristic of leaky tumor tissue (Nevozhay, 2007). Nanocarriers as drug delivery systems are designed to enhance the pharmacological and therapeutic properties of conventional medicaments. Incorporating a drug compound molecule into nanocarriers can protect it from degradation and also offers the possibility of targeting and controlled release. 
Due to their small dimensions, nanocarriers are able to cross the blood-brain barrier and act at the cellular level. Despite the existence of many therapeutic agents based on nanoparticles, only a small amount of nanoparticle drugs is undergoing preclinical studies [12].

\section{Bibliography}

1. Sanvicens N., M. Pilar Marco, Multifunctional nanoparticles -properties and prospects for their use in human medicine, Trends in Biotechnology 2008 Vol.26 No.8

2. Klębowski B, Depciuch J, Parlińska-Wojtan M, Baran J. Applications of Noble Metal-Based Nanoparticles in Medicine. Int J Mol Sci . 2018; 19 (12): 4031. Opublikowano 13 grudnia 2018 r. Doi: 10.3390 / ijms 19124031

3. Graczyk A, Pawlowska R, Jedrzejczyk D, Chworos A. Gold Nanoparticles in Conjunction with Nucleic Acids as a Modern Molecular System for Cellular Delivery. Molecules. 2020;25(1):204. Published 2020 Jan 3. doi:10.3390/molecules25010204

4. Harrison B. S., Atala A., Carbon nanotube applications for tissue engineering, Biomaterials, Vol. 28, 2007, 344-353

5. Gutwein LG, Webster TJ: Affects of alumina and titania nanoparticulates on bone cell function., American Ceramic Society 26 the Annual Meeting Conference Proceedings 2003 in press.

6. Ma J, Wong H, Kong LB, Peng KW: Biomimetic processing of nanocrystallite bioactive apatite coating on titanium. Nanotechnology 2003, 14:619-623

7. Salata OV, Applications of nanoparticles in biology and medicine, Journal of Nanobiotechnology 2004, 2, 1-6

8. Wang A. Z, Langer R, Farokhzad O. C., Nanoparticle Delivery of Cancer Drugs, Annual Review of Medicine, 2012, Vol. 63: 185-198

9. Roy I, Ohulchanskyy TY, Pudavar HE, Bergey EJ, Oseroff AR, Morgan J, Dougherty TJ, Prasad PN: Ceramic-based nanoparticles entrapping water-insoluble photosensitizing anticancer drugs: a novel drug-carrier system for photodynamic therapy. J Am Chem Soc 2003, 125:7860-7865.

10. Cao YC, Jin R, Nam JM, Thaxton CS, Mirkin CA: Raman dyelabeled nanoparticle probes for proteins. Journal of the American Chemical Society 2003, 125:14676-14677.

11. Bamrungsap S.; Zhao Z.; Chen T.; Wang L.; Li C.; Fu T., ; Tan W., Nanotechnology in Therapeutics, Nanomedicine. 2012;7(8):1253-1271

12. Wilczewska A. Z, Niemirowicz K., Markiewicz K.H, Car H., Nanoparticles as drug delivery systems, Pharmacological Reports, 2012, 64, 1020-1037 